\title{
On the Identification of Preisach Measures
}

\author{
Matthew E. Shirley and Ram Venkataraman \\ Texas Tech University Department of Mathematics and Statistics, Lubbock, TX 79409-1042.
}

\begin{abstract}
The phenomenon of hysteresis is commonly encountered in the study of magnetic materials. The Preisach operator and its variants have been successfully used in the modeling of a physical system with hysteresis. In an application, one has to determine a density function for the Preisach operator using the input-output behavior of the system at hand. In this paper, we describe a method for numerically determining an approximation of the density function when there is not enough experimental data to uniquely solve for the density function. We also present numerical results where we estimate an approximate density function from data published in the literature for a magnetostrictive actuator and an electro-active polymer.
\end{abstract}

Keywords: Hysteresis, Preisach Operator, Density function Identification, Constrained least squares, Smart materials, EAPs

\section{INTRODUCTION}

The phenomena of nonlinear hysteresis has been well documented in magnetism and electricity. Hysteresis comes from a Greek term meaning "to lag behind," and describes a relationship between inputs and outputs of a certain system. A system with scalar inputs and outputs is said to exhibit rate-independent hysteresis if: (a) the outputs of the system do not depend on the rate at which the input is applied and (b) the outputs of the system at a certain time, depend on the past history of the input function. Though dynamical systems exhibit the "memory" requirement (b), they do not exhibit the "rate independence" requirement (a).

The Preisach operator is a mathematical tool that has been used to model the phenomena of hysteresis for many years. ${ }^{1,2}$ Part of what is needed to describe the Preisach operator for a particular system is a density function defined for certain parameters. In the past, several researchers have addressed the problem of identifying the Preisach density function. Mayergoyz ${ }^{2}$ first described a method to identify the density function in the proof of his characterization theorem. However, this method cannot be used in practice when the output signal is corrupted by noise, as it involves a differentiation of the output signal. Banks, Kurdila and Webb ${ }^{3}$ address the problem of convergence of the approximate measures identified through experiments to the true density function, while Hoffman and Meyer ${ }^{4}$; Hoffmann and Sprekels ${ }^{5}$; and Galinaitis and Rogers ${ }^{6}$ discuss the use of the linear least squares method to identify the Preisach measure. In Venkataraman, Tan and Krishnaprasad, a constrained linear least squares method is used to explicitly constrain the approximate density function to be positive. ${ }^{7}$ Galinaitis, Joseph and Rogers ${ }^{8}$ (and the references therein) use a parametrization of the density functions in terms of basis functions in order to reduce the number of parameters to be identified. However this method has some severe problems that are described in that paper.

In this paper, we consider the identification of the Preisach density function when there is not sufficient experimental data. Mayergoyz's representation theorem ${ }^{2}$ yields a sufficiency condition on the input-output signal, so that a complete identification of the measure can be achieved. However, in practice, this would involve a very large amount of data that has to be processed to obtain the density function. There is a need for a method that uses limited information to obtain an approximation of the density function. Another instance of the problem of insufficient data is encountered when one considers a fine discretization of the input signal in order to obtain a smaller approximation error (this is described in more detail in Section 4). What is needed is a method that utilizes all the available "information" in the experimental data to obtain the best approximation of the actual density function.

We cast the identification problem as a constrained minimization problem (the details are in Section 3):

$$
\min \frac{1}{2}\|A X-Y\|^{2} \quad \text { subject to } \quad X \geq 0
$$


where $A$ and $Y$ are computed using the input and output signals respectively, and $X$ is unknown. Our methodology involves the identification of the nullspace of the matrix $A^{T} A$ and obtaining a solution in its orthogonal complement. In Section 2, we briefly describe the Preisach operator and describe the problem in Section 3. In Section 4 we describe the results of our numerical experiments for a magnetostrictive actuator and an electroactive polymer.

\section{THE PREISACH OPERATOR}

The Preisach operator has been used to model hysteresis in many applications. In the following section, we will define the Preisach operator, and discuss its properties.

Consider a relay, $R_{\beta, \alpha}$ (see figure 1 ), which at any given time is at one of two states: +1 or -1 . Consider an input function $\mathrm{u}(\mathrm{t})$ for the relay. Define the output $v(t)$ to be ${ }^{1}$ :

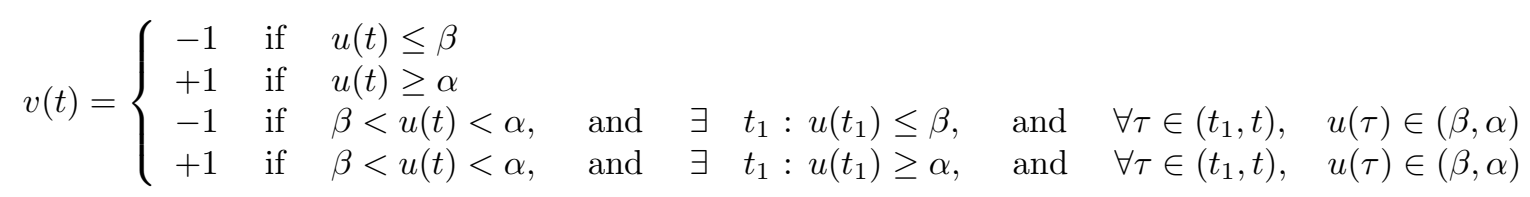

This gives us the relation shown in figure 1.

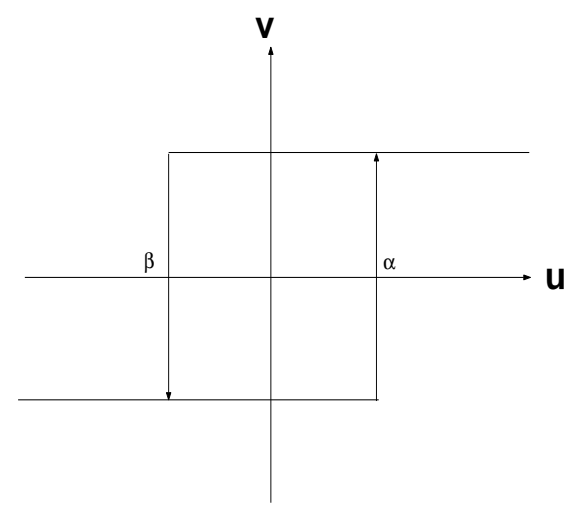

Figure 1. Input-output relationship from (1)

To construct the Preisach operator when the input is $u(\cdot)$, denote $v_{\beta, \alpha}(\cdot)=R_{\beta, \alpha}[u](\cdot)$. The Preisach operator's input is $u(\cdot)$, and the output is given $b^{8}$ :

$$
y(t)=\iint_{\alpha \geq \beta} \mu(\beta, \alpha) R_{\beta, \alpha}[u](t) d \beta d \alpha
$$

where $\mu(\beta, \alpha)$ is a continuous density function with compact support. It can be shown that when $u(\cdot)$ is piecewise monotone, then so is $y(\cdot){ }^{1}$

Denote the set of relays with value +1 at time $t$ as $S_{+}(t)$, and the relays with value -1 at time $t$ as $S_{-}(t)$. The curve separating $S_{+}$and $S_{-}$(henceforth referred to as a memory curve) corresponds to the state of a dynamical system in the following sense ${ }^{1}$ :

- If we knew the memory curve at time $t$ and the input function over the interval $[t, T]$, then the memory curve at time $T$ can be computed uniquely; 
- Equation (2) can be considered as a map from the space of memory curves to the set of output values; in other words, the memory curve at a time $t$ yields a unique output value at that time.

Thus we can define a Preisach operator to be a map $\Gamma: C_{p m}[0, T] \rightarrow C_{p m}[0, T]$, where $u(\cdot) \mapsto y(\cdot)$. It has been shown that $\Gamma$ has several useful mathematical properties ${ }^{1}$ : (a) Lipschitz continuity, (b) regularity, (c) invertibility. In addition it has the so-called "congruency", and "wiping-out" properties (please refer the Mayergoyz for an excellent description ${ }^{2}$ ). The wiping out property is observed in magnetic materials and "smart" actuators such as those based on piezoelectricity and magneto-striction. This makes the Preisach operator a valuable mathematical tool for researchers in smart structures.

\section{THE PROBLEM}

When using the Preisach operator to model a physical system, it is necessary to find the density function $\mu(\beta, \alpha)$, that models the physical phenomenon. We have to determine $\mu$ from experiments, by observing outputs $y(\cdot)$ that correspond to inputs $u(\cdot)$. What we would like is an approximation of the density function $\mu$ to put into the Preisach operator which fits the current input and output data.

The proof of Mayergoyz's representation theorem ${ }^{2}$ yields a way to exactly compute the density function $\mu$, but we need continuous inputs and no sensor noise. The second condition cannot be assumed from experimental data and so we need another method to determine $\mu$. Due to the experimental limitations, we have to discretize the input values. This leads to a discretization of the Preisach plane. This procedure is described in what follows.

To implement this, the first thing to do is to discretize the input function $u(t)$. We divide the time interval $[0, T]$ into nodes $t=t_{0}, t_{1}, \ldots, t_{n}$, where $t_{0}=0$, and $t_{n}=T$. Assume that a tolerance $d$ for the input has been specified, and use this to round the input values to the nearest multiple of the tolerance. Thus $u\left(t_{i}\right) ; i=0, \ldots, n$ takes values in the set $N d$, where $N \in \mathbb{I}$, a subset of the set of $\mathbb{Z}$.

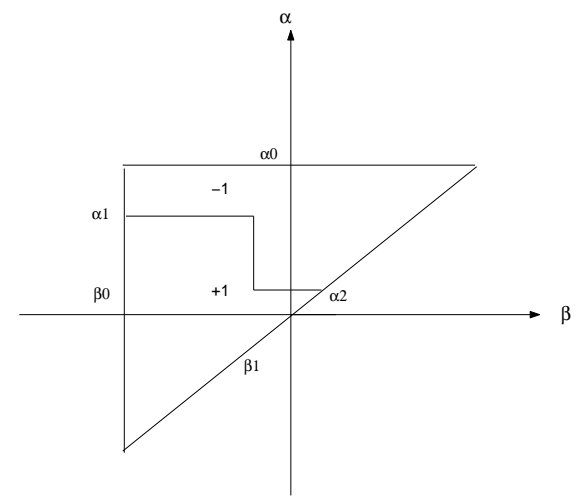

Figure 2. Input increasing to $\alpha_{1}$, then decreasing to $\beta_{1}$, and then increasing to $\alpha_{2} \in\left(\beta_{1}, \alpha_{1}\right)$

Once the input is discretized, the Preisach plane, defined to be $\{(\alpha, \beta) \mid m \leq \alpha, \beta \leq M\}$, (where $m$ is the minimum value of $\alpha$ and $\beta$, and $M$ is the maximum value of $\alpha$ and $\beta$ ) is dicretized accordingly. Round the lower bound $m$ down to the nearest accuracy from the tolerance, $\hat{m}$, and round the upper bound $M$ up to the nearest accuracy of the tolerance, $\hat{M}$.

The next step is to divide the $\alpha$ and $\beta$ axes into pieces of length $d$. On each axis start with $\hat{m}$ and make a division at $\hat{m}+d$, the next at $\hat{m}+2 d$, and so on. When this is completed, there will be $\left(\frac{\hat{M}-\hat{m}}{d}\right)$ intervals along both axis, and the plane will be divided into a finite number of partitions (see figure below). Let $D \subset P$ be defined so that our input is restricted to $P \backslash D$, then we only need to discretize the compliment of $D$. In the following, we denote the contribution to the output due to the set $D$ to be a constant $c$. 
The result is that if we plot a discretized input function $u(t)$ the input values will match up along the grid formed on $P$. This is because we used the same tolerance for both $u(t)$ and $P$. From here, we would like to assign a number to label each piece of $P$. There will be $\left[\frac{\hat{M}-\hat{m}}{d}-1\right]\left[\frac{\hat{M}-\hat{m}}{d}\right] / 2$ total partitions.

Now, to approximate $\mu$ at some point $p$ we will find the density measure of a neighborhood $R$ of $p$, then assume that $R$ has a uniform density. Then, we can let $\mu(p)=\mu(R)$. Let $(n-1)$ be the number of partitions of the plane, and let us assume a uniform density in each partition. Let $x_{i}$ represent the density of the i'th section of the plane, $\forall i=1, \ldots,(n-1)$. To make things easier, put the entries $\bar{x}_{i}$ into a vector $\bar{X}$, so that

$$
\bar{X}=\left[\begin{array}{c}
\bar{x}_{1} \\
\vdots \\
\bar{x}_{n-1}
\end{array}\right]
$$

Now the problem becomes finding an approximation for the vector $\bar{X}$. We find $\bar{X}$ using the input and output data.

Consider the Preisach plane with a memory curve. Let this be the state at time $t$. Since we partitioned the Preisach plane from discretization of the input, each point on the memory curve has $(\alpha, \beta)$ coordinates $\left(M_{1} d, M_{2} d\right)$, for some $M_{1}, M_{2} \in \mathbb{I}$. The result is that the memory curve lines up with the plane's partition.

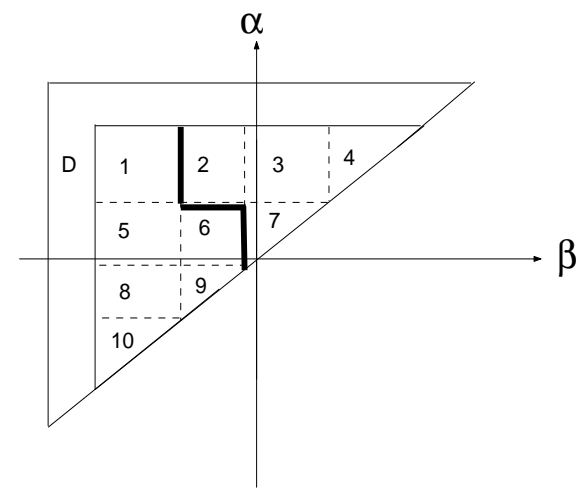

Figure 3. Partitioned plane with a memory curve

Let $\bar{X}_{1}$ be the set of $\bar{x}_{i}$ below the memory curve. Thus $\bar{X} \backslash \bar{X}_{1}$ is the set of $\bar{x}_{i}$ above the memory curve. Then, we would like to say that the output $y(t)$ at time $t$ is approximated by

$$
y(t) \approx \sum_{m \in \bar{X}_{1}} \bar{x}_{m}+\sum_{m \in \bar{X} \backslash \bar{X}_{1}}(-1) \bar{x}_{m}+c
$$

In the case of Figure 3 we would have

$$
y \approx(+1)\left[\bar{x}_{1}+\bar{x}_{5}+\bar{x}_{6}+\bar{x}_{8}+\bar{x}_{9}+\bar{x}_{10}\right]+(-1)\left[\bar{x}_{2}+\bar{x}_{3}+\bar{x}_{4}+\bar{x}_{7}\right]+c
$$

Why can we divide the Preisach plane, and approximate the density at each piece? It turns out that as the partition gets finer (converging to a point), the density approximation converges to the actual density at each point in $P$. This is stated in the following proposition.

Before we start the proposition, we need the following definition.

Definition 3.1. The diameter of a set $A$, which is a subset of an Euclidean space, (notated diam $(A)$ ), is defined as

$$
\operatorname{diam}(A)=\max \{|p-q|: \forall p, q \in A\}
$$


Proposition 3.1. Let $P$ represent the Preisach plane, and let $P_{n}$ represent the pieces of the discretized plane. Furthermore, let $y$ be the output for the Preisach operator, then $\sum_{m \in X_{1}} x_{m}+\sum_{m \in X \backslash X_{1}}(-1) x_{m} \rightarrow y$ as $\max \left\{\operatorname{diam}\left(P_{n}\right): P_{n} \in P\right\} \rightarrow 0$.

For a proof please refer to Banks, Kurdila, and Webb. ${ }^{3}$

During an experiment, the outputs might get corrupted by sensor noise, and this might cause the measurements $y_{i}$ to be inexact. To model this, we write the output at time $t$ as:

$$
y(t)=\sum_{m \in \bar{X}_{1}} \bar{x}_{m}+\sum_{m \in \bar{X} \backslash \bar{X}_{1}}(-1) \bar{x}_{m}+c+\epsilon
$$

where the $\epsilon$ term takes care of the approximation error, and the error due to noise.

Define a row vector $A_{1}$ to be $A_{1}=\left[\begin{array}{llll}\delta_{1} & \cdots & \delta_{n-1}\end{array}\right]$, where the i'th element is

$$
\delta_{i}=\left\{\begin{array}{cl}
1 & \text { if } \bar{x}_{i} \in \bar{X}_{1} \\
-1 & \text { if } \bar{x}_{i} \in \bar{X} \backslash \bar{X}_{1}
\end{array}\right.
$$

Using this new vector, the output at this state is

$$
y\left(t_{1}\right)=\bar{A}_{1} \bar{X}+c+\epsilon=\left[\begin{array}{lll}
\delta_{1} & \cdots & \delta_{n-1}
\end{array}\right]\left[\begin{array}{c}
\bar{x}_{1} \\
\vdots \\
\bar{x}_{n-1}
\end{array}\right]+c+\epsilon=\sum_{i=1}^{n-1} \delta_{i} \bar{x}_{i}+c+\epsilon
$$

For easier notation let $A_{1}=\left[\begin{array}{ll}\bar{A}_{1} & 1\end{array}\right]$ and $X=\left[\begin{array}{c}\bar{X} \\ c\end{array}\right]$, then (6) becomes $y\left(t_{1}\right)=A_{1} X$

If we do this for $\mathrm{m}$ instances of time then we get $\mathrm{m}$ row vectors $\left\{A_{j}: j=1, \ldots, m\right\}$, with each $A_{j}=$ $\left[\begin{array}{lllll}\delta_{j 1} & \cdots & \delta_{j(n-1)} & 1\end{array}\right]$. If we denote $y\left(t_{j}\right)=y_{j}$, we also have $\mathrm{m}$ outputs $\left\{y_{j} \approx A_{j} X: j=1, \ldots, m\right\}$. The system looks like the following:

$$
\left\{\begin{array}{lllll}
y_{1} & = & A_{1} & X & +\epsilon_{1} \\
y_{2} & = & A_{2} & X & +\epsilon_{2} \\
\vdots & & \vdots & & \\
y_{m} & = & A_{m} & X & +\epsilon_{m}
\end{array}\right.
$$

Let $\left[\begin{array}{c}y_{1} \\ \vdots \\ y_{m}\end{array}\right]=Y,\left[\begin{array}{c}A_{1} \\ \vdots \\ A_{m}\end{array}\right]=A$, and $\left[\begin{array}{c}\epsilon_{1} \\ \vdots \\ \epsilon_{m}\end{array}\right]=\epsilon$

Then, we have the linear equation

$$
Y=A X+\epsilon
$$

We need is a way to solve for $X$ that will best fit the data, but at the same time keep $x_{i} \geq 0$, since $X$ represents a density function.

We would like to minimize the function

$$
f(X)=\frac{1}{2}\|A X-Y\|^{2}
$$

where 


$$
A: \mathbb{R}^{n} \rightarrow \mathbb{R}^{m}, \quad X \in \mathbb{R}^{n}, \quad Y \in \mathbb{R}^{m}, \quad \text { and } \quad \operatorname{rank}(A)=m
$$

with the inequality constraint $g(X)=X \geq 0$.

The Lagrange multiplier theorem yields the existence of a $\lambda \in \mathbb{R}^{n}$, such that the necessary conditions for $X$ to minimize (8) are, ${ }^{9}$

1.

$$
\lambda_{i}=0 \text { when } X_{i} \neq 0
$$

2.

$$
\lambda_{i} \geq 0 \text { when } X_{i}=0
$$

3. The augmented function

$$
\begin{aligned}
\bar{f}(X) & =f(X)-\lambda^{T} g(X) \\
& =f(X)-\lambda^{T} X
\end{aligned}
$$

satisfies

$$
\frac{\partial \bar{f}(X)}{\partial X}=X^{T} A^{T} A-Y^{T} A-\lambda^{T}=0
$$

We used the MATLAB routine "quadprog" to solve this problem and the results are described in Section 4 (please see Figures 4 and 5).

\subsection{Insufficient experimental data}

The method described earlier works very well when the experimental input-output data is in a form to yield an $m \times n A$ matrix with rank $n$. However, if we do not have such an situation (which can easily arise by choosing a finer discretization of the input), we must reformulate the problem. In the following, we consider an even more general problem where the rank of $A$ is less than $\min \{m, n\}$ :

$$
\text { minimize } f(X)=\frac{1}{2}\|A X-Y\|^{2} ; \quad \operatorname{rank}(A)<\min \{m, n\},
$$

with the conditions:

$$
\begin{aligned}
& A X=Y+\epsilon \\
& X_{i} \geq 0, \quad \forall i=1, \ldots, n
\end{aligned}
$$

Now, we need to minimize (13), and $\frac{1}{2}\|X\|^{2}$. This problem can be tackled in two ways. The first method involves a regularization:

$$
\text { minimize } \quad g(X)=\frac{\alpha}{2}\left\|X^{2}\right\|+\frac{1}{2}\|A X-Y\|^{2}, \quad \text { subject to } \quad X_{i} \geq 0, \quad \forall i=1, \ldots, n, \quad \text { where } \quad \alpha>0
$$

The second method involves a singular value decomposition of $A$ so that we now perform the minimization on an orthogonal subspace to the nullspace of $A^{T} A$. We found that the regularization approach is unstable numerically, as the choice of $\alpha$ affects the solution greatly. We now describe the second solution method below.

Let $\operatorname{rank}(A)=q<\min \{m, n\}$. If we perform a singular value decomposition on $A^{T} A$, then we get $A^{T} A=U S V^{T}$, where $S$ is an $n \times n$ diagonal matrix with $\operatorname{rank} q<n$. If we eliminate the rows and columns of $S$ that are zero and the corresponding columns of $U$ and $V$, then we end up with a $q \times q$ diagonal matrix that 
we call $\hat{S}$, and two $n \times q$ matrices $\hat{U}$ and $\hat{V}$. As $A^{T} A$ is symmetric, $\hat{U}$ and $\hat{V}$ are identical. This fact we exploit in the following.

We have $\hat{V} \hat{S} \hat{V}^{T}=A^{T} A$, where $\hat{V}^{T} \hat{V}$ is a $q \times q$ identity matrix. Now if we let $X=\hat{V} Z$ then the Problem (8) becomes:

$$
\text { minimize } f(Z)=Z^{T} \hat{S} Z-Y^{T} A \hat{V} Z,
$$

subject to the constraint $g(Z)=\hat{V} Z \geq 0$. Then once we have a minimizer $Z^{*}$ to the above problem, then $X^{*}=\hat{V}^{T} Z^{*}$ solves Problem (8). The constrained minimization problem (16) can be solved by using the MATLAB routine "quadprog".

In section 4, we considered a finer discretization of the input which led to the case considered in this subsection. The results can be seen in Figures 6 and 7 .

\section{NUMERICAL RESULTS}

The following experiments were performed in a MATLAB environment on a PC running a $1.6 \mathrm{GHz}$ Athlon Processor, with a 1 GB RAM. We used experimental data from a commercial magnetostrictive actuator from Venkataraman, Tan and Krishnaprasad ${ }^{7}$ in our initial numerical experiments. There, a Preisach operator was used to model the average magnetic field versus magnetization characteristic. In the experiment, the magnetic field input took values between $10 \mathrm{~A} / \mathrm{m}$ and $410 \mathrm{~A} / \mathrm{m}$. The output values were measured in $O e$. We discretized the Preisach plane into intervals of $20 \mathrm{~A} / \mathrm{m}$. We used input data to create an $A$ matrix, whose dimensions were $1036 \times 211$. The rank of $A$ was 211 , and so we used the MATLAB command "quadprog" which solves (8). The time taken for the computation of $X$ was 6.076 seconds, and the maximum difference $\max _{1 \leq i \leq 1036}\left|A_{i} X-Y(i)\right|=$ $6.626 \times 10^{4} O e$, (where $A_{i}$ denotes the $i-t h$ row of $A$ ). This error can be compared against the maximum and minimum values of $Y$ that were $7.708 \times 10^{5} \mathrm{Oe}$ and $2.143 \times 10^{5}$ Oe respectively. A comparison between the $A X$ and $Y$ can be seen in figure 5 . The discretized density obtained can be seen in figure 4 .

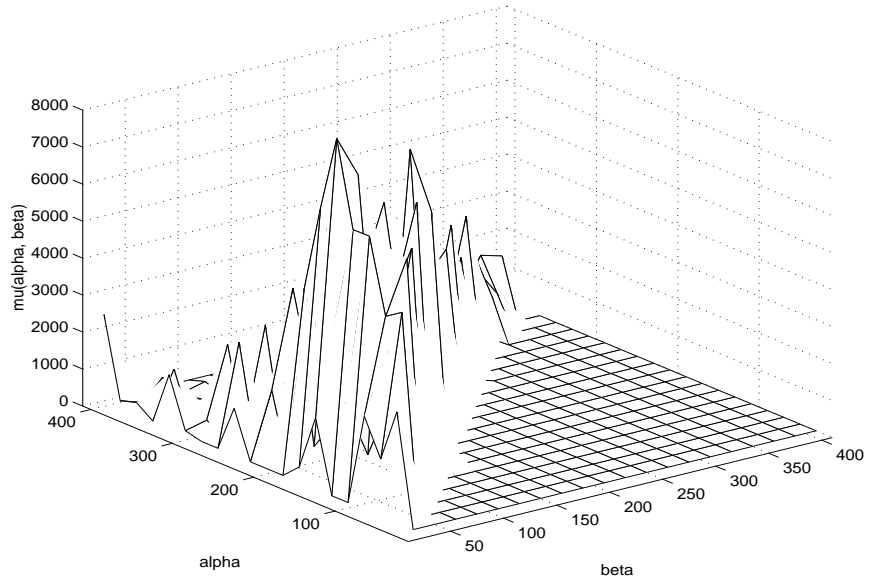

Figure 4. The Preisach density calculated with an input magnetic field discretization of $20 \mathrm{~A} / \mathrm{m}$.

In order to reduce the error $\max _{i}\left|A_{i} X-Y(i)\right|$, we discretized the Preisach plane into intervals of $10 \mathrm{~A} / \mathrm{m}$. The input data led to a $1036 \times 821 A$ matrix. However, this time the $\operatorname{rank}(A)=516$ so we had to use the method described in Subsection 3.1. We used the MATLAB command "svd" to obtain a singular value decomposition for $A^{T} A$, and then used the "quadprog" command to obtain the minimizer $X_{*}$. The discretized density function obtained can be seen in figure 6 . It took 616 seconds for the computation, but $\max _{i}\left|A_{i} X-Y(i)\right|=3.206 \times 10^{4}$. As expected, the comparison between $A X$ and the experimental output showed a better match than the discretization 


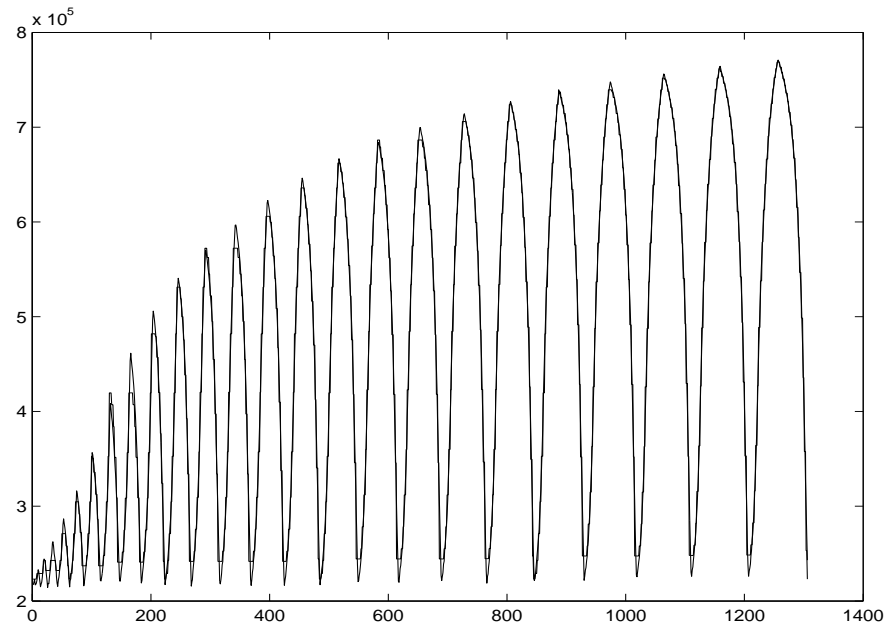

Figure 5. The approximation to the output magnetization calculated with an input magnetic field discretization of $20 \mathrm{~A} / \mathrm{m}$, compared against data from experiment.

level of 20, the results can be seen in figure 7. Finally, Figure 8 shows the magnetic field versus magnetization characteristic for the magnetostrictive actuator obtained as a result of the identification experiment.

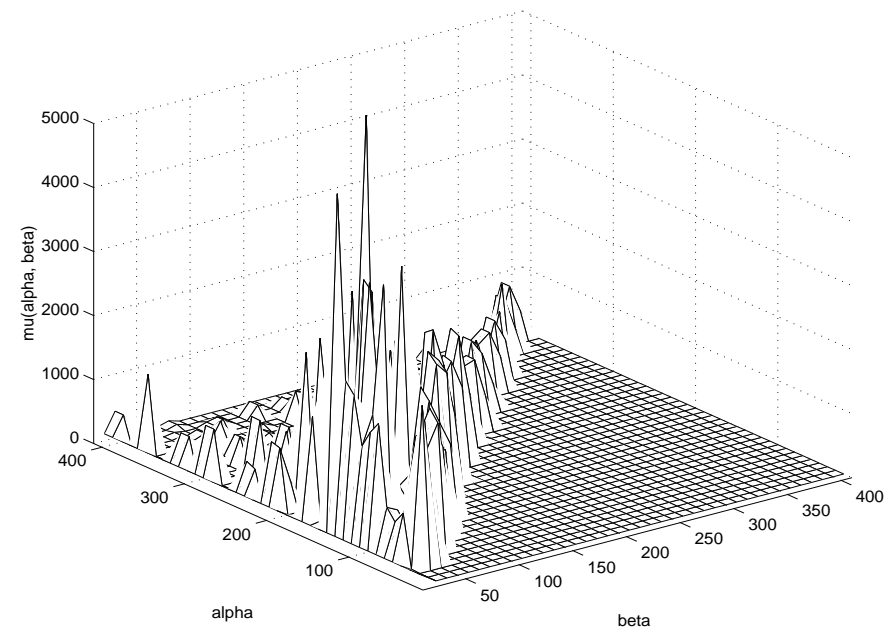

Figure 6. The Preisach density calculated with an input magnetic field discretization of $10 \mathrm{~A} / \mathrm{m}$.

The next numerical experiments conducted were on data published by Petchsuk and Chung ${ }^{10}$ on an electroactive polymer (VDF/TrFE/HFP $(55.17 / 42.35 / 2.46)$ terpolymer 121$)$ at $42^{\circ} C$. This polymer exhibits significant hysteresis in its Electric Displacement vs Electric Field characteristic. The discretization was of the Electric Field was chosen to be $12.5 \mathrm{MV} / \mathrm{m}$ The identified Preisach density function can be seen in Figure 9. The comparison between the fitted data and the experimental data obtained from Petchsuk and Chung ${ }^{10}$ can be seen in Figure 10.

\section{CONCLUSIONS}

In this paper, we have described a method to compute (an approximation of) the Preisach density function when there is insufficient experimental data. This is a situation that is frequently encountered in practice. Previous 


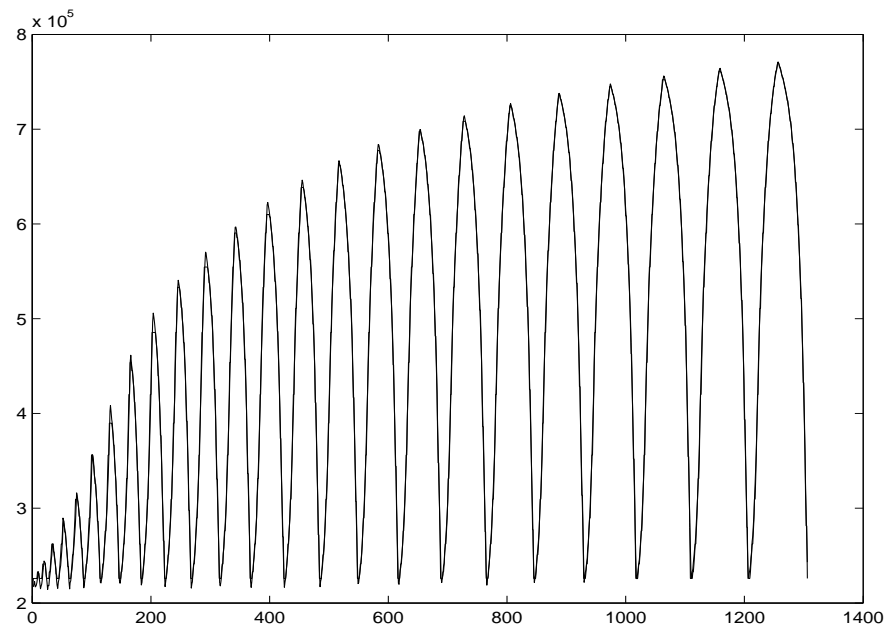

Figure 7. The approximation to the magnetization output calculated with an input magnetic field discretization of $10 \mathrm{~A} / \mathrm{m}$, compared against data from experiment.

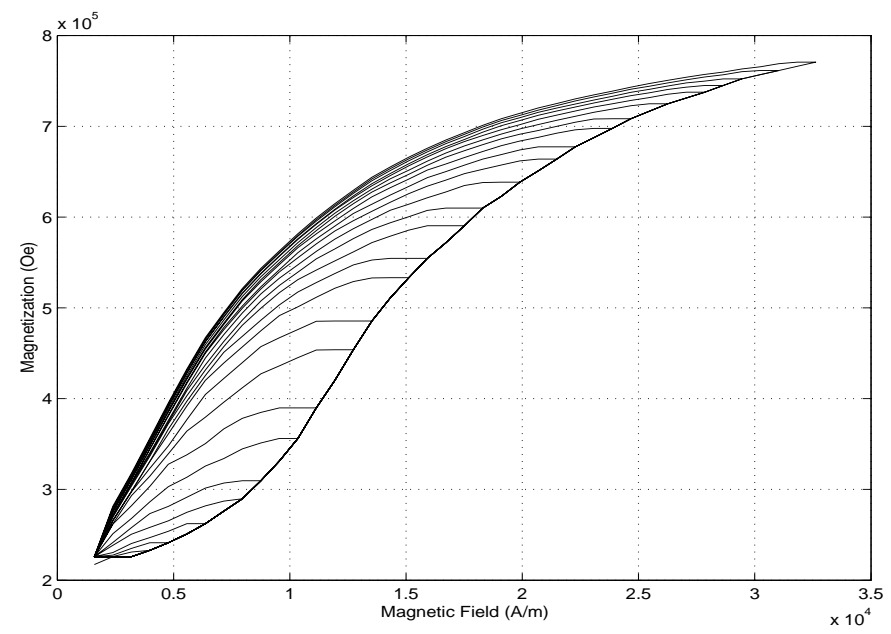

Figure 8. Fitted Magnetic field versus Magnetization characteristic.

methods assumed the availability of sufficient data for the identification to be performed, or considered a very coarse grid on the Preisach plane leading to a poor approximation of the actual density function. We describe a method that utilizes all the information present in the input-output data to obtain the best approximation possible. We also present numerical results using experimental input-output data, where we compare the results of our identification with published data.

\section{ACKNOWLEDGMENT}

We gratefully acknowledge Prof. Chung and the Materials Research Society, for allowing us to use Figure 4 from Petchsuk and Chung ${ }^{10}$ in this manuscript.

\section{REFERENCES}

1. M. Brokate and J. Sprekels, Hysteresis and Phase Transitions. Springer, 1996.

2. I. Mayergoyz, Mathematical Models of Hysteresis. Springer, 1991. 


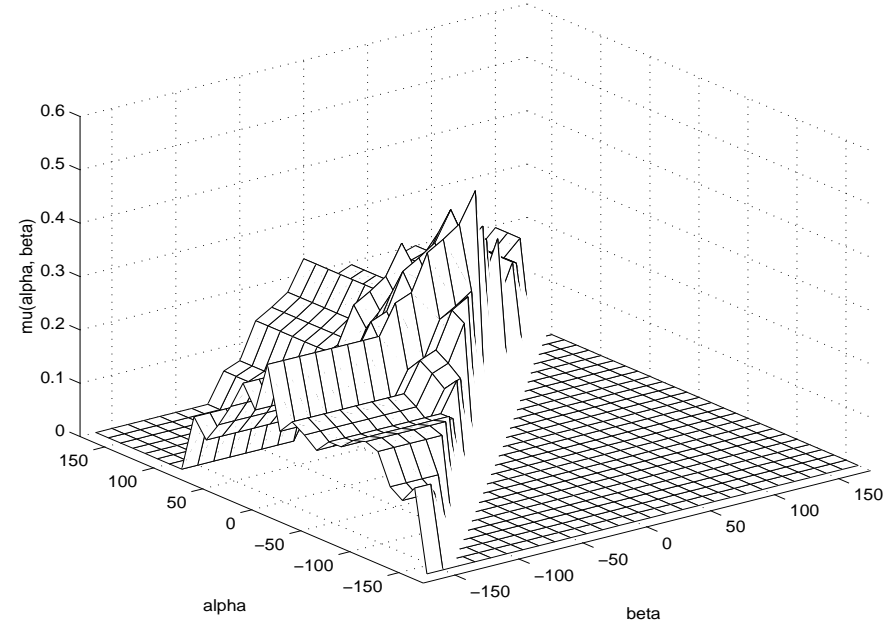

Figure 9. The Preisach density calculated for a VDF/TrFE/HFP electro-active polymer.

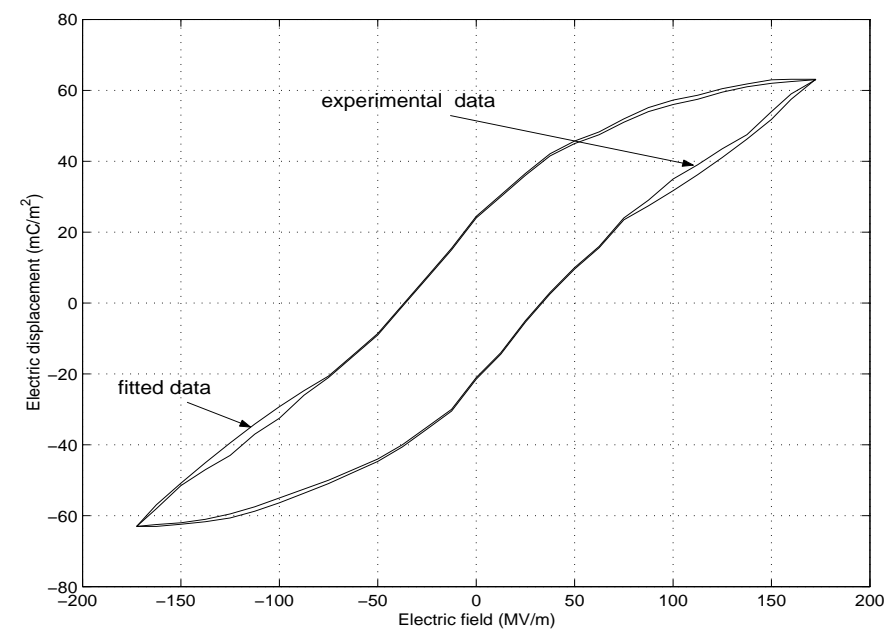

Figure 10. Comparison between fitted and actual charge versus electric field data for a VDF/TrFE/HFP electro-active polymer.

3. H. T. Banks, A. J. Kurdila, and Webb, "Identification of hysteretic control influence operators representing smart actuators - part i: Formulation," Tech. Rep. CRSC-TR96-14, Center for research in scientific computation, North Carolina State University, 1996.

4. K. H. Hoffmann and G. H. Meyer, "A least squares method for finding the preisach hysteresis operator from measurements," Numer. Math., vol. 55, pp. 695-710, 1989.

5. K. H. Hoffmann and J. Sprekels, "Identification of hysteresis loops," Jour. Computational Pysics, vol. 78, pp. 215-230, 1988.

6. W. Galinaitis and R. C. Rogers, "Control of a hysteretic actuator using inverse hysteresis compensation," in Mathematics and Control in Smart Structures, Smart Structures and Materials 1998 (V. V. Varadhan, ed.), vol. 3323, pp. 267-277, Mar. 1998.

7. R. Venkataraman, X. Tan, and P. Krishnaprasad, "Control of hysteresis: Theory and experimental results," in Smart Structures and Materials 2001: Modeling, Signal Processing, and Control in Smart Structures (V. Rao, ed.), vol. 4326, Mar. 2001. 
8. W. S. Galinaitis, D. Joseph, and R. C. Rogers, "Parameter identification for preisach models of hysteresis," in Proceedings of DETC 2001 ASME Design Engineering Technical Conferences, pp. 267-277, Mar 2001.

9. D. G. Luenberger, Optimization by Vector Space Methods. Wiley, 1968.

10. A. Petchsuk and T. Chung, "Synthesis and electric property of VDF/TrFE/HFP TerPolymers," in Electroactive Polymers (EAP) (Y. B. Q. M. Zhang, Takeo Furukawa and J. Scheinbeim, eds.), vol. 600, pp. 53-60, Nov-Dec 1999. 